\title{
Printed Large-area Photovoltaic Modules based on Small-molecules with Different Alkyl Terminal
}

\section{Chains}

Youn-Jung $\mathrm{Heo}^{1}$, Bogyu Lim ${ }^{2,3, *}$,Yen-Sook Jung ${ }^{1}$, Kyeongil Hwang ${ }^{1}$, Jueng-Eun Kim ${ }^{1}$, Donmin Lee ${ }^{1}$, Dae-Hee Lim ${ }^{1,4}$, Jong Mok Park ${ }^{3}$, and Dong-Yu Kim ${ }^{1, *}$

${ }^{1}$ School of Material Science and Engineering, Research of Institute of Solar and Sustainable Energies, Gwangju Institute of Science and Technology, Gwangju 500-712, Republic of Korea

${ }^{2}$ Future Technology Research Center, Corporate R\&D, LG Chem/LG Science Park, 30, Magokjungang 10-ro, Gangseo-gu, Seoul, 07796, Republic of Korea

${ }^{3}$ Green Fine Chemical Research Center, Advanced Convergent Chemistry Division, Korea Research Institute of Chemical Technology $\quad$ (KRICT), $45 \quad$ Jongga-ro, Junggu, Ulsan, 44412, Republic of Korea

${ }^{4}$ Energy Materials Research Center, Korea Research Institute of Chemical Technology (KRICT), 141, Gajeong-re, Yuseong-gu, Daejeon, 34114, Republic of Korea 
Corresponding Authors*: E-mail: bglim@krict.re.kr, kimdy@gist.ac.kr 
Table S1. Photovoltaic parameter of slot-die coated solar cells with different amount of DIO additives

\begin{tabular}{cccccc}
\hline Small molecule & Volume of additive (DIO) & $\mathbf{V}_{\text {oc }}(\mathrm{V})$ & $\mathbf{J}_{\mathbf{s c}}\left(\mathbf{m A ~} \mathbf{~ c m}^{-2}\right)$ & $\mathbf{F F}$ & $\mathrm{PCE}(\%)$ \\
\hline LGC-D073 & $0 \%$ & 0.89 & 9.2 & 38.3 & $3.13(2.96)$ \\
$3 \%$ & 0.84 & 9.7 & 59.6 & $4.93(4.48)$ \\
$6 \%$ & 0.85 & 9.8 & 61.9 & $5.17(5.08)$ \\
$9 \%$ & 0.84 & 11.3 & 62 & $5.82(5.56)$ \\
& $12 \%$ & 0.81 & 9.64 & 62 & $4.84(4.70)$ \\
\hline LGC-D023 & $0 \%$ & 0.86 & 6.61 & 51.7 & $2.95(2.72)$ \\
& $3 \%$ & 0.85 & 9.49 & 65.7 & $5.33(5.10)$ \\
& $6 \%$ & 0.85 & 8.27 & 58.9 & $4.13(3.95)$ \\
& $9 \%$ & 0.86 & 7.74 & 58.4 & $3.93(3.60)$ \\
& $12 \%$ & 0.88 & 4.74 & 52.3 & $2.17(2.05)$ \\
\hline
\end{tabular}



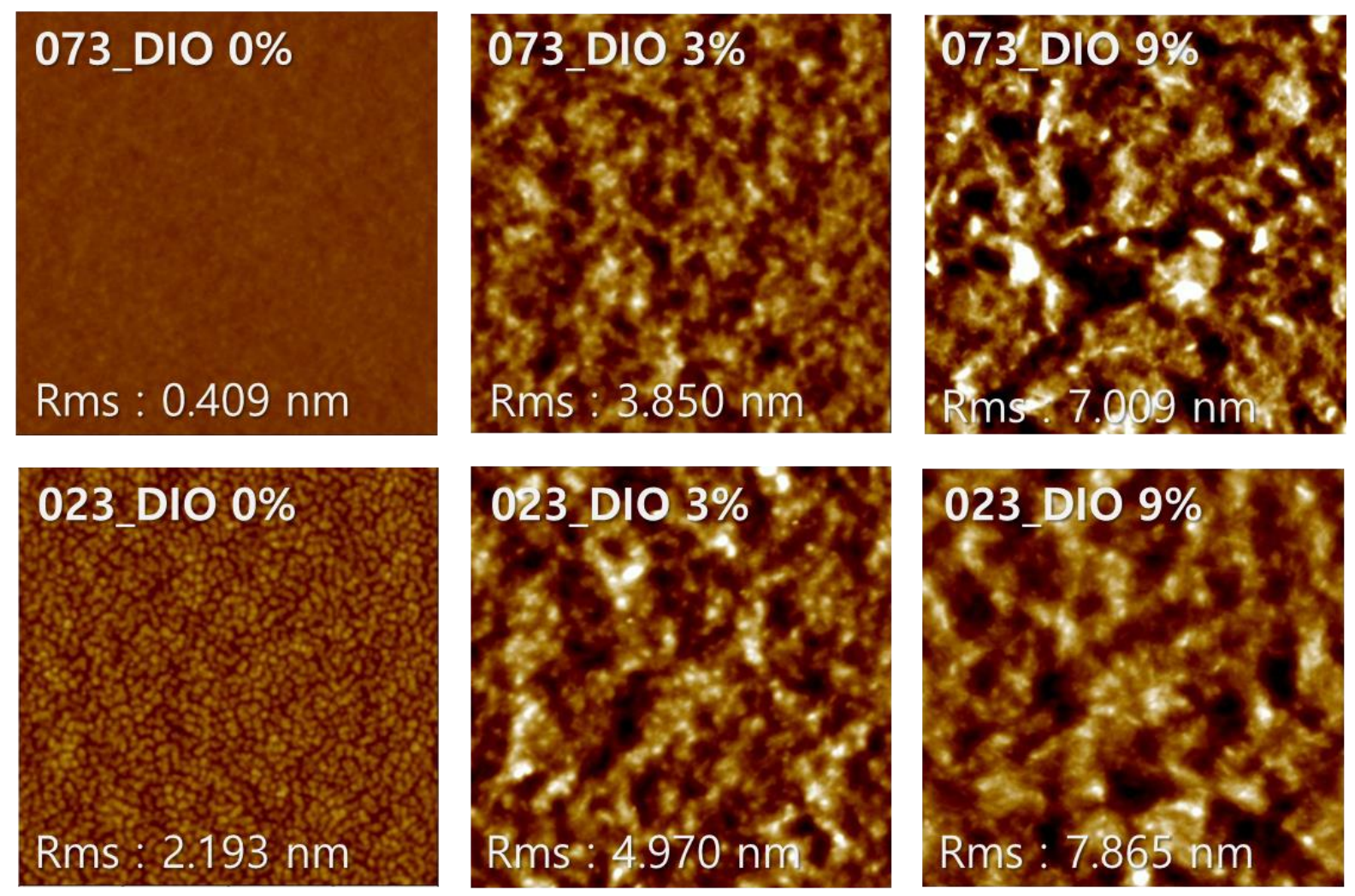

Figure S1. AFM images of slot-die coated thin films. 

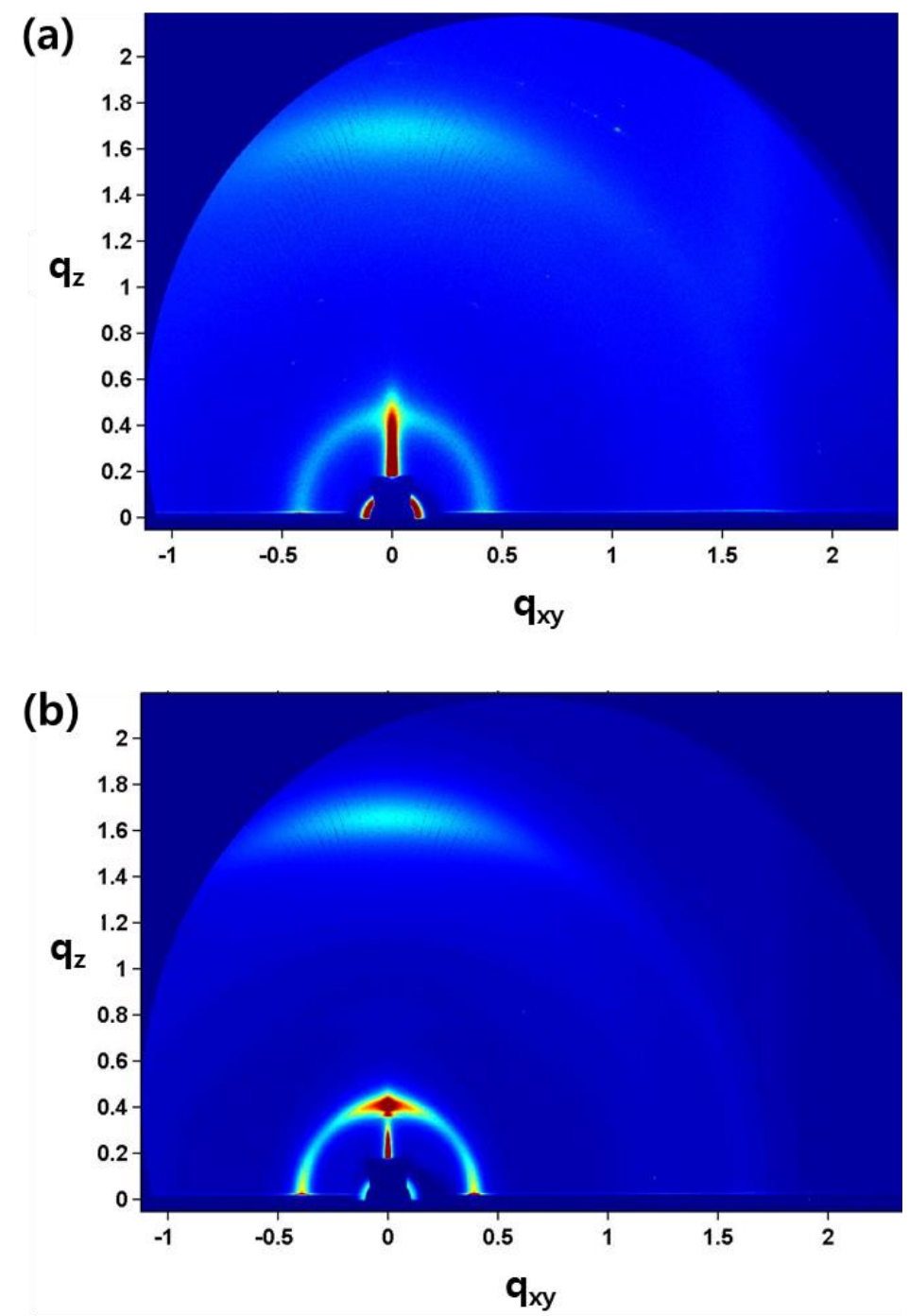

Figure S2. 2D GIXD patterns for pure films of (a) LGC-073 and (b) LGC-023 small molecules. 
Table S2. Crystallographic information of slot-die coated thin films based on the small molecules

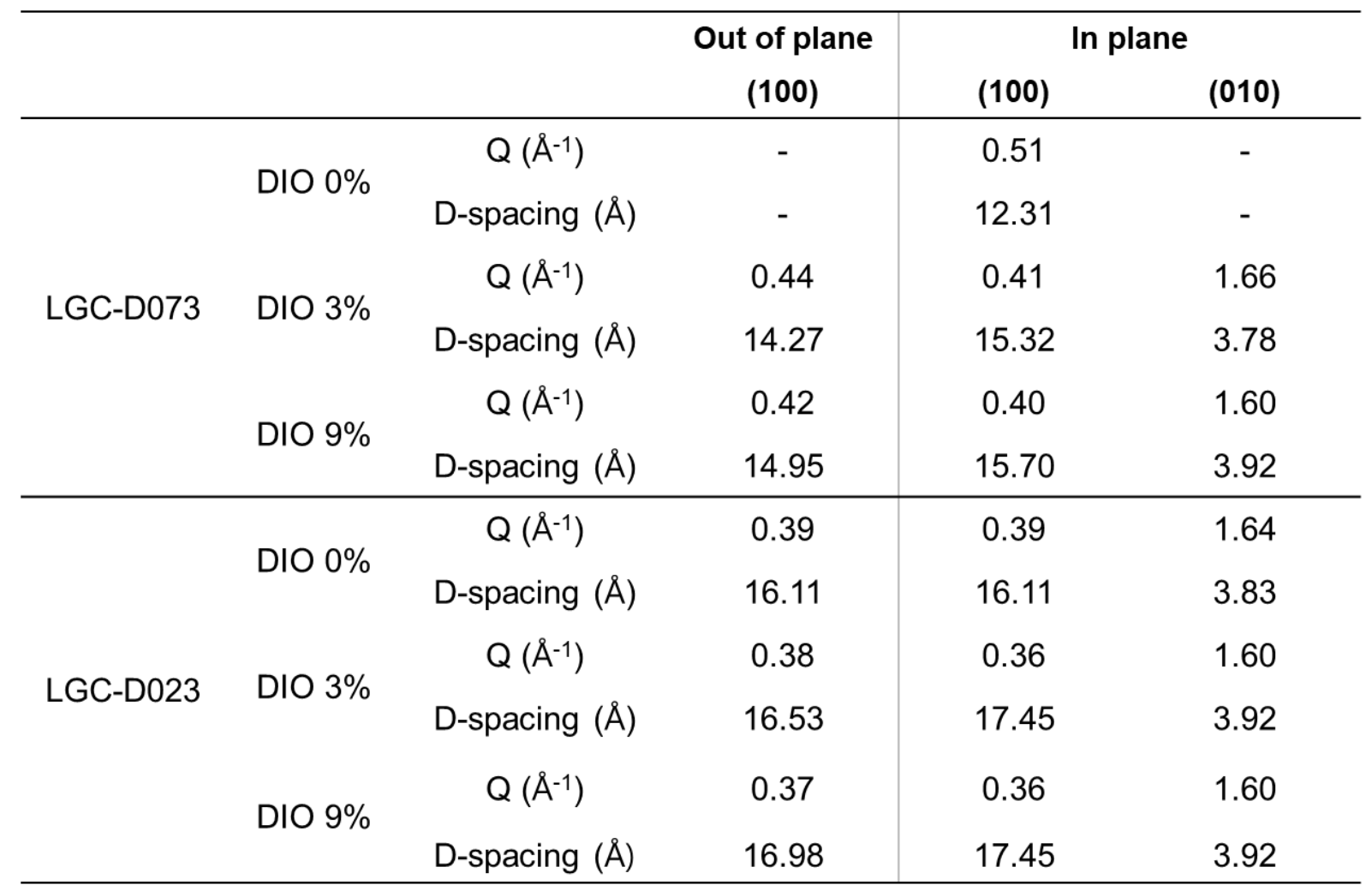


(a)

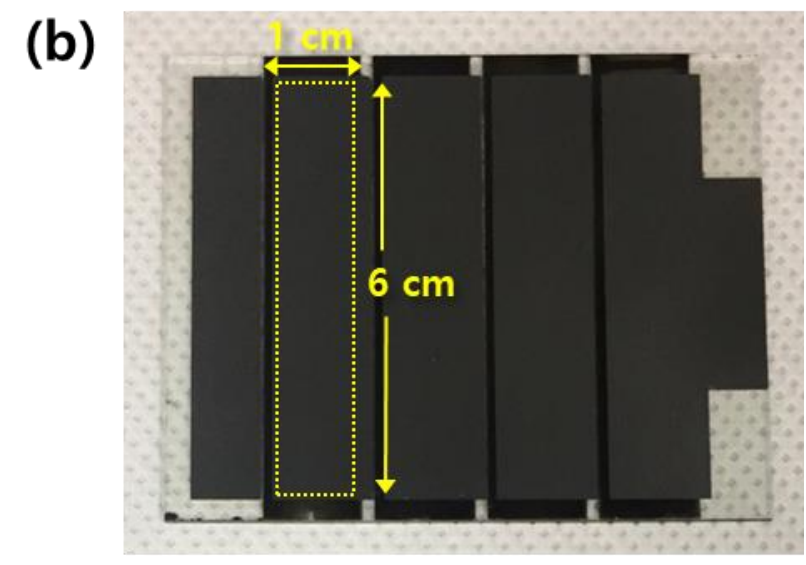

Active area $=0.1 \mathrm{~cm}^{2}$

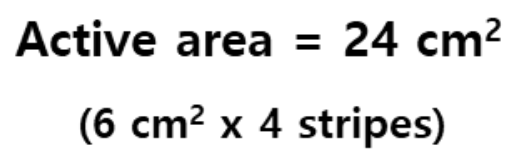

Figure S3. Photographic of (a) small-area single-cell and (b) large-area photovoltaic modules. The geometrical FF of large-area photovoltaic modules is calculated to be $50 \%$. (The geometrical FF is percentage of the active area of $24 \mathrm{~cm}^{2}$ divided by substrate area of $48 \mathrm{~cm}^{2}$.) 
Table S3. Photovoltaic parameters of slot-die printed large-area modules

\begin{tabular}{ccccc}
\hline Photovoltaic modules & $\mathbf{V}_{\text {oc }}(\mathbf{V})$ & $\mathbf{J}_{\text {sc }}\left(\mathbf{m A ~ \mathbf { ~ c m } ^ { - 2 }}\right)$ & FF & PCE(\%) \\
\hline LGC-D073 & $3.30(3.11)$ & $3.10(2.72)$ & $54(48.50)$ & $5.50(4.17)$ \\
LGC-D023 & $3.47(3.33)$ & $2.28(2.10)$ & $57(55.06)$ & $4.53(3.90)$ \\
\hline
\end{tabular}




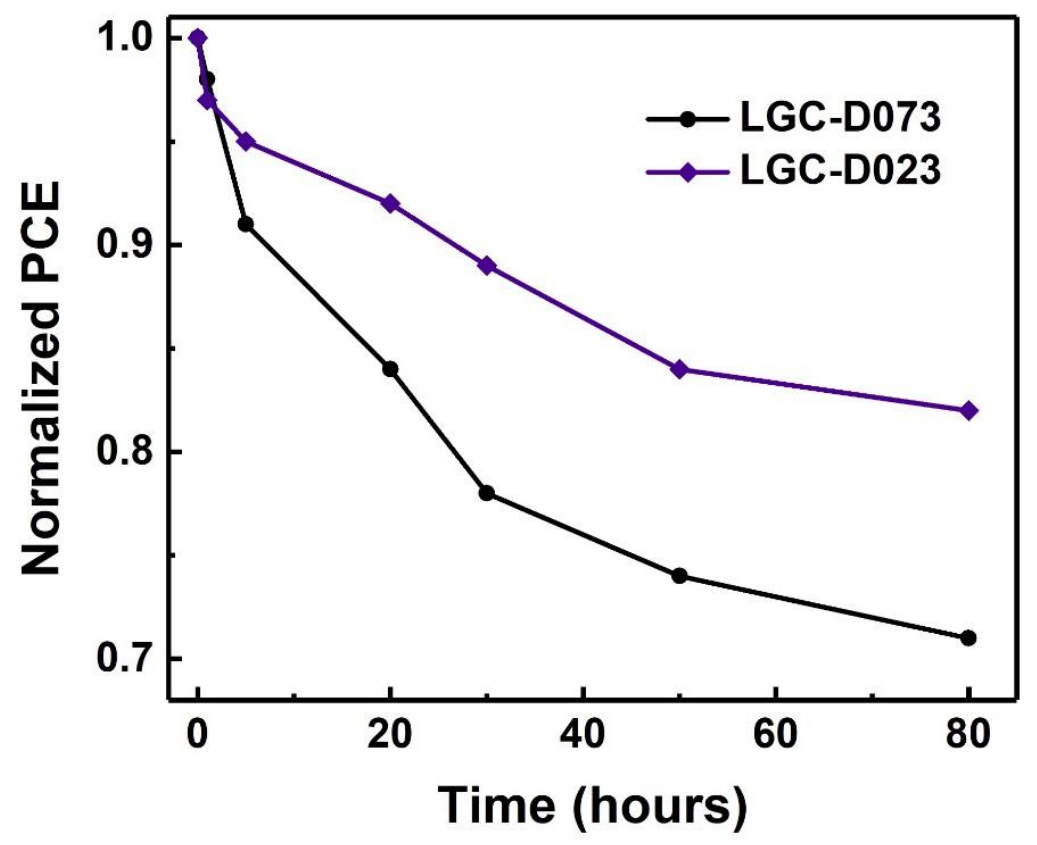

Figure S4. Normalized power conversion efficiency of slot-die coated devices as a function of storage time in $\mathrm{N}_{2}$ atmosphere. The relative lower stability of LGC-D073 will be attributed to the higher volume of DIO additive. 


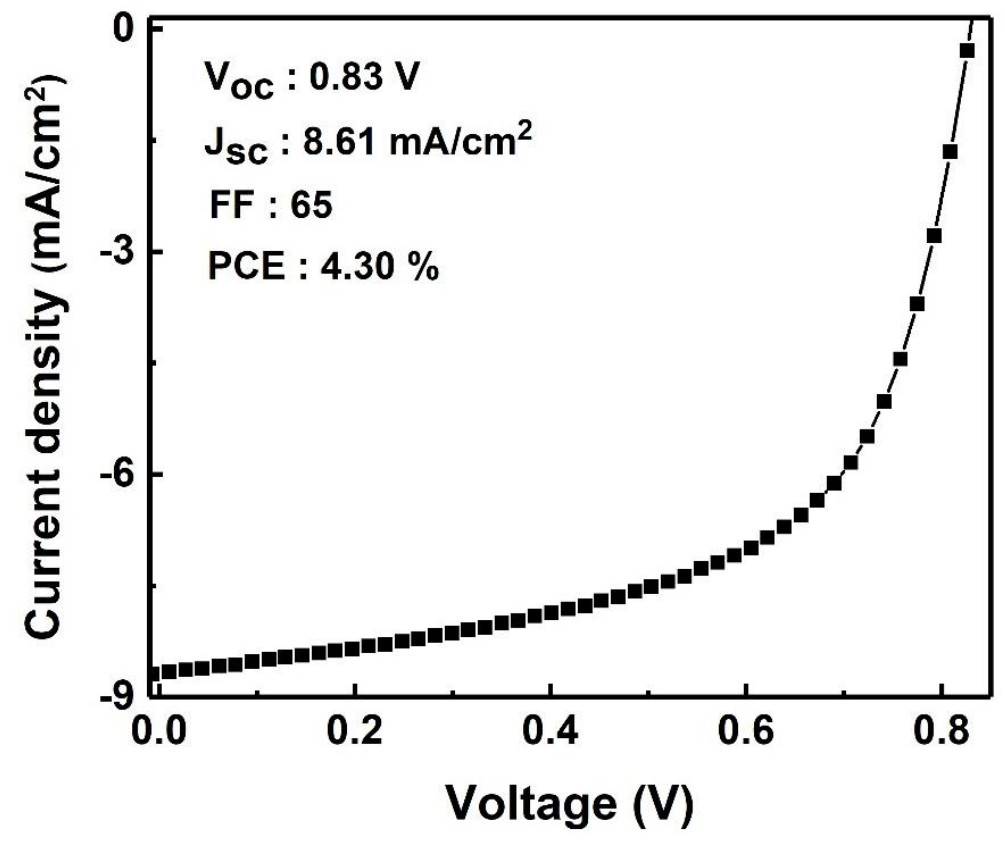

Figure S5. The J-V curves of slot-die coated devices processed from non-halogenated solvent, xylene. Due to the better solubility of LGC-D023, we just tried to fabricate the solar cells by using the only LGC-D023. The quite low efficiency of slot-die coated solar cells will be attributed to the relatively poor solubility for xylene compared to the chlorobenzene. 